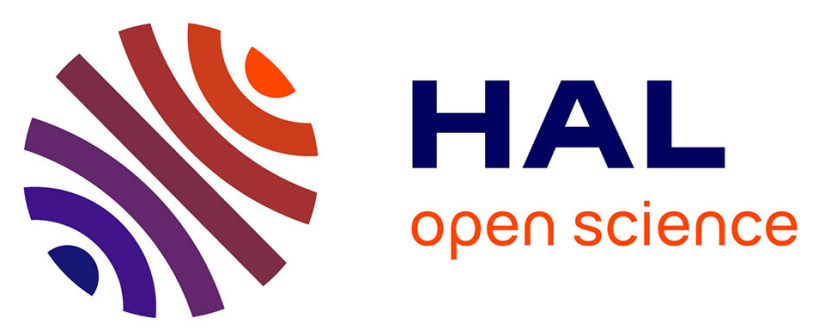

\title{
LES STRATÉGIES DE DIFFUSION PUBLIQUE DE L'INFORMATION : L'EXEMPLE DE LA FÉDÉRATION SPORTIVE DE NATATION DANS LA MÉDIATISATION DES ÉVÉNEMENTS
}

\author{
Marie Ouvrard-Servanton, Audrey de Céglie
}

\section{To cite this version:}

Marie Ouvrard-Servanton, Audrey de Céglie. LES STRATÉGIES DE DIFFUSION PUBLIQUE DE L'INFORMATION : L'EXEMPLE DE LA FÉDÉRATION SPORTIVE DE NATATION DANS LA MÉDIATISATION DES ÉVÉNEMENTS. Les Enjeux de l'information et de la communication, 2012, 13/2, p.73 à p.90. hal-01444128

HAL Id: hal-01444128

https://hal-amu.archives-ouvertes.fr/hal-01444128

Submitted on 24 Jan 2017

HAL is a multi-disciplinary open access archive for the deposit and dissemination of scientific research documents, whether they are published or not. The documents may come from teaching and research institutions in France or abroad, or from public or private research centers.
L'archive ouverte pluridisciplinaire HAL, est destinée au dépôt et à la diffusion de documents scientifiques de niveau recherche, publiés ou non, émanant des établissements d'enseignement et de recherche français ou étrangers, des laboratoires publics ou privés. 


\title{
Les strałégies de diffusion publique de l'information : I'exemple de la fédération sportive de natation dans la médiatisation des événements
}

Article inédit. Mis en ligne le 20 féurier 2013.

\author{
Marie Ouvrard-Servanton et Audrey De Ceglie \\ Université, LSIS - UMR CNRS 6168. \\ Toulouse 3. \\ Plan \\ L'information institutionnelle et la communication médiatique \\ Les questions, les hypothèses et le corpus de recherche \\ La compétition sportive : un artefact public \\ L'espace et le temps de l'événement sportif \\ L'événement sportif : simulacre ou réalité? \\ L'influence et la visibilité du cadre institutionnel \\ Une analyse entre omission et polémique \\ Conclusion \\ Références bibliographiques
}

Marie Ouvrard-Servanton est docteur en Sciences de l'Information et de la Communication. Aix-Marseille

Audrey De Ceglie est maître de conférences en Sciences de l'Information et de la Communication, LERASS,

\section{Résumé}

L'objectif de cet article est d'étudier l'influence de la fédération française de natation (FFN) dans la diffusion des événements sportifs par l'instauration de règles (temps de qualification, réglementations, etc.) ou d'artefacts communicationnels (coupes, championnats, etc.) et d'étudier comment ces événements sportifs sont relayés pour le public par une communication médiatique de l'information institutionnelle à travers un média de presse écrite. Une analyse comparative de l'information institutionnelle diffusée par la fédération via Internet, un questionnaire soumis à la Direction Technique Nationale et un corpus journalistique sont utilisés pour mettre en évidence ce qui influence l'événement de communication à forte représentation dans l'espace public.

Mots clés : information institutionnelle, média presse écrite, artefact, événement sportif

\section{Abstract}

The objective of this article is to study the influence of the French Swimming Federation $(\mathrm{FFN})$ in the diffusion of sportive events through promulgation of rules (qualification times, rules, etc.) or communication artifacts (challenges, championships, etc.) and to study how the sporting events are relayed to the public by a communication media style of the institutional information through the print media. A comparative analysis of the information disseminated by the swimming federation over the Internet, an enquiry submitted to the National Technical Director and a journalistic corpus, are used to highlight what influences the communication event that is strongly represented in the public space.

\section{Resumen}

El objetivo de este articulo es de estudiar la influenza de la federación francés de natación (FFN) en la difusión de los eventos deportivos a través de la reglas (tempos de 
cualificaciones, reglamentos, etc.) o de los artefactos comunicacionales (copas, campeonatos, etc.) y de estudiar como estos eventos están transmitidos para el publico por la comunicación mediática de la información a través el media escrito. Una análisis de la información difundida por la federación francés de natación a través Internet, de un cuestionario a la Dirección Técnica Nacional y de un corpus periodístico se utiliza para resaltar lo que influye en el caso de la comunicación con una alta representación en el espacio público.

\section{L'INFORMATION INSTITUTIONNELLE ET LA COMMUNICATION MEDIATIQUE}

Sur son site Internet, la Fédération Française de Natation (FFN) publie les informations d'ordre réglementaire qui encadrent l'organisation et le déroulement des compétitions sportives. En vue des Jeux Olympiques de 2012, elle rend disponible les critères de sélection pour les épreuves de Natation Course au travers d'un document écrit, accessible sur son site, nommé Critères de Sélection Natation Course Octobre 2011 à décembre 2012. Entre autre, ce document montre la feuille de route des compétitions sportives, nationales et internationales, de natation qui aboutiront aux Jeux Olympiques 2012. Dès lors, cette information institutionnelle devient-elle potentiellement publique ? Comme le démontre Jocelyne Arquembourg à partir des travaux de John Langhsaw Austin, «le fait de rendre public constitue un acte de langage particulier, plus proche d'un acte illocutoire ${ }^{1}$ que d'un acte perlocutoire ${ }^{2}$, et dont l'efficacité est garantie par l'existence d'un appareillage sociotechnique et politique à la fois vaste et complexe. Cet acte ne peut prendre effet que pour autant que des communautés d'action sont concernées, en l'occurrence, des publics.» (Arquembourg, 2010, p.184). Or quelle communauté est concernée par les critères de sélection de la Natation Course ? Dans un premier temps, seuls les spécialistes de la natation (entraineurs, nageurs, membres de l'encadrement, de la Fédération, de clubs, journalistes, chercheurs, etc.) y ont recours. Cependant, les compétitions de natation ne restent pas des actions entre spécialistes. De plus, «l'existence publique en tant qu'objet de préoccupation commune pour une communauté d'action, est alors autre chose que l'existence «quelque part».» (Arquembourg, 2010, p.184). Nous pouvons admettre que ces compétitions doivent leur existence au cadre réglementaire officiellement publié sans lequel la compétition ne pourrait avoir lieu.

Par ailleurs, les compétitions de natation dépassent amplement le cadre des institutions et des spécialistes de ce sport. Mis à part le public présent dans les gradins, un public plus vaste s'intéresse à la compétition des courses de natation des Jeux Olympiques par le biais de la diffusion médiatique de la télévision, de la radio et de la presse écrite, voire du moyen hybride qu'est Internet. Selon Médiamétrie, environ neuf millions de téléspectateurs ont regardé les finales des courses de natation des JO de Londres 2012 sur une des chaines de France Télévision. Ces médias effectuent une mise en mémoire de l'événement qui laisse des traces telles qu'elles ont été enregistrées à un moment donné.

\footnotetext{
${ }^{1}$ Qui accomplissent quelque chose en le disant

${ }^{2}$ Qui effectuent quelque chose par le fait de dire
} 
Bien qu'ayant chacune un public, l'information institutionnelle de la FFN et la communication médiatique sont deux types de diffusion qui n'ont pas le même public (Lavoinne, 1997, p.24). Le premier type s'adresse à un public restreint bien qu'il soit potentiellement accessible à tous alors que le deuxième est destiné intentionnellement à un public de masse. Via le site Internet de la FFN, l'encadrement réglementaire est consulté essentiellement par des spécialistes alors que le journal L'Équipe que nous avons choisi pour notre corpus, est à portée de tous.

\section{LES QUESTIONS, LES HYPOTHESES ET LE CORPUS DE RECHERCHE}

Que fait ressortir la presse écrite des informations institutionnelles publiées par la FFN qui encadrent les compétitions de natation, dans la création de l'événement sportif ?

De cette question principale peuvent se décliner les questions de recherche suivantes :

Q1 : Quel rôle joue le cadre réglementaire en tant qu'information institutionnelle dans l'avènement de l'événement sportif ?

Q2 : La presse écrite participe-t-elle au réalisme de l'événement sportif ?

Q3 : Dans la communication médiatique opérée par la presse écrite, le fait de rendre visible ou invisible le cadre réglementaire comme une information émanant de l'institution contribue-t-il à l'avènement de l'événement sportif et communicationnel aux yeux du public?

A partir de ces questions, nous avons émis les hypothèses suivantes afin de pouvoir vérifier leur validité :

H1 : Le cadre réglementaire érige de fait la compétition sportive comme un artefact qui, sans lui, n'aurait pas lieu d'être.

H2 : La presse écrite a besoin du cadre réglementaire pour rendre réaliste l'événement sportif.

H3 : Le média presse écrite s'empare de ce qui se passe "hors cadre » en omettant (en rendant invisible) la source ou une partie de l'information institutionnelle pour favoriser une communication événementielle.

Pour tracer le chemin de la médiatisation et montrer ce qui se passe entre "afficher publiquement/publier le cadre réglementaire des compétitions » et «rendre public l'événement de la compétition ", nous avons limité notre étude à la consultation des textes publiés sur le site de la FFN, à la consultation d'un Directeur Technique National par le biais d'un questionnaire et à la sélection d'un corpus dans le journal L'Equipe, sur les deux compétitions de natation permettant la sélection des nageurs pour les Jeux Olympiques de Londres 2012.

Le Directeur Technique National (DTN) de natation de 2000 à 2008 est un acteur déterminant car il a assumé pleinement le rôle assigné par le projet fédéral (de la FFN) en étant l'initiateur d'une nouvelle réglementation pour les critères de sélection et en la diffusant autant à l'intérieur de la Fédération qu'auprès des médias. Le projet fédéral qui est analysé dans un rapport de l'INSEP ${ }^{3}$ met en exergue les deux types de responsabilité du

\footnotetext{
${ }^{3}$ Rapport d'étude - Analyse de l'activité du Directeur Technique National et de la Direction Technique Nationale pour concevoir la formation des futurs DTN - MOA : Direction des sports - MOE : INSEP (Institut
} 
DTN. D’une part, le DTN assume des responsabilités internes à la Fédération concernant le respect des valeurs, des objectifs et des règles qui contribuent à définir les relations internes au sein de la Fédération. D'autre part, il assume des responsabilités externes vis-à-vis d'acteurs tels que le ministère chargé des sports, d'autres ministères, les médias, les organismes privés, les organismes pour le développement des pratiques, etc. En tant que DTN, Claude Fauquet a donc géré les Olympiades de 2000, 2004 et 2008 avec la nouvelle réglementation des critères de sélection des nageurs français.

Dans L'Équipe, nous avons sélectionné une série d'articles écrits à propos des grandes échéances pour la natation française en vue des olympiades. En France, le journal L'Équipe a une position hégémonique quasi monopolistique dans la presse quotidienne sportive. De ce fait, L'Équipe est un support de référence pour relayer la communication des compétitions sportives. Le choix d'un corpus issu de ce journal a été motivé par le fait que nous voulions récolter des écrits afin de comprendre si et comment la réglementation passant par l'écrit pour être publiée, était retranscrite par la presse écrite.

La discipline sportive, la natation, a été choisie car elle est peu sujette à la subjectivité d'arbitres (comme le sont les sports d'équipe, notamment) ou de juges (comme le sont la natation synchronisée, le patinage artistique, la gymnastique, etc.) concernant les résultats. $\mathrm{Au}$ moment où le nageur touche à l'arrivée le bord de la piscine, le temps est enregistré électroniquement au centième de seconde près. Les courses sont dorénavant filmées, et plus spécifiquement les départs. Ces éléments laissent peu de place à l'aléatoire. Comme le règlement laisse peu de place à l'interprétation, nous mettrons en lumière de façon plus précise les écarts opérés par la presse écrite.

\section{LA COMPETITION SPORTIVE : UN ARTEFACT PUBLIC}

Les «Championnats de France de Natation» (CF) sont une compétition et un événement en natation. Nous considérons l'artefact « $\mathrm{CF}$ de Natation» comme communicationnel car, en plus d'être décidé et encadré par la FFN, il est relayé par les organisateurs auprès des clubs, des pôles sportifs et des médias (notamment via la presse papier, Internet ou audiovisuelle). Il est défini comme un effet attendu soit «le résultat d'une action", (Agostinelli, 4/2009), attendu par l'art, soit «une activité conduite et considérée comme un ensemble de règles à observer; un ensemble d'activités humaines créatrices d'ouvrages contenant les préceptes, les règles d'une discipline » (Agostinelli, 4/2009), comme le sont les compétitions de natation dans le cadre de la FFN avant même que les courses aient lieu. Dès lors, le cadre et la dénomination de la compétition sportive, artefact de communication, effet communicationnel de l'art, acquièrent un caractère public, devenant visible/lisible à un grand nombre de personnes.

Avant que ces Championnats aient lieu, l'annonce de cet artefact communicationnel induit un effet de représentation, montrant ce qui n'est pas encore visible dans l'action, si ce n'est à travers le langage ou l'image (Goody, 2006, p.45). Un effet communicationnel de l'art est produit : "CF de Natation Course ", tel que cadré par la FFN, au travers de la réglementation (un ensemble de règles) éditée en tant qu'information institutionnelle, bien avant que la compétition ne se manifeste. Ce cadrage influence notamment les

[suite de la note]

National du Sport, de l'Excellence et de la Performance (Paris), Rapport rédigé sous la direction de Philippe Fleurance, Unité Etudes, Ingénierie et Innovation, Mars 2008 
nageurs dans leurs activités et conditionne leur participation aux JO 2012 de Natation Course.

$\mathrm{Au}$ moment où une compétition est lancée, l'appellation « $\mathrm{CM}$ » ou « $\mathrm{CF}$ », devient un artefact qui va créer un événement communicationnel avant que l'événement sportif ne se déroule. La compétition est prévue par la FFN en interne bien avant que l'artefact de la compétition sportive soit lancé officiellement. Un temps et un espace ont été dédiés à cet événement artefactuel et une série de petits événements internes (prises de décision, annonces des décisions, formation de couple entraineurs/entrainés, arrivée de nouveaux sponsors, etc.) ont eu lieu avant que l'événement ne se déclenche à l'extérieur. Plus qu'une hypothèse (H1), c'est une condition : le cadre réglementaire décidé, nommé et prenant la forme d'une information institutionnelle érige de fait la compétition sportive comme un artefact qui, sans lui, n'aurait pas lieu d'être. L'événement de la compétition sportive ne peut avoir lieu qu'autour d'un artefact.

\section{L'ESPACE ET LE TEMPS DE L'EVENEMENT SPORTIF}

Pour avancer dans la vérification des hypothèses suivantes et comprendre quels rôles jouent l'encadrement institutionnel de la compétition et la presse écrite dans l'avènement de l'événement sportif, nous devons préalablement définir ce que nous entendons par événement. Dans un second temps, nous développons la méthodologie permettant de vérifier nos hypothèses et de répondre in fine à nos questions, en la reliant aux éléments qui définissent l'événement de communication, à la façon dont nous abordons les mots écrits et les signes comme représentation de l'action et à des indicateurs issus de l'anthropologie des sciences adaptés à l'analyse de la communication.

Dans des travaux précédents, nous avons approché la notion d'événement de communication (Ouvrard-Servanton, 2010) pour en extraire son lien avec l'artefact, l'espace et le temps. Après avoir établi que la communication est une somme d'expériences où les acteurs humains et non humains échangent à travers le langage, l'événement (Deleuze, 1969) s'effectue autour d'un artefact (Agostinelli, 2003), dans un espace lisse ou strié (Deleuze \& Guattari, 1980) et dans un temps nommé Aiôn, terme grec pour définir un présent étendu incluant ce qui est déjà passé et encore futur, en opposition à chronos. L'espace strié est un espace sédentaire, codé, organisé, structuré et ordonné où le but et les règles sont importants, comme l'est la réglementation dans notre exemple. L'espace lisse est un espace nomade, variable, en mouvement, non délimité où les frontières ne sont pas définies, où la direction et le trajet sont plus importants que le but et où le territoire est mouvant. Cet espace admet tout ce qui va déborder. Dans ces travaux, nous avons démontré que l'événement de communication s'effectue dans les interstices de l'espace strié et qu'un excès de "striage » et d'encodage pouvait étouffer l'action. Si l'on supposait que strictement tous les nageurs avec les meilleurs temps de sélection soient les vainqueurs des épreuves lors des compétitions et que cette hypothèse se vérifie inlassablement, les compétitions de natation, par exemple, auraient-elles la même portée publique et médiatique ?

Un nouvel enjambement s'opère dans le passage de relais entre la diffusion de l'information règlementaire publiée par la FFN, artefact communicationnel, et la production d'un événement sportif. Dans son ouvrage, La Peur des représentations, Jack Goody pose le problème général de la mimesis tout en admettant que « la représentation a de toute évidence une importance centrale dans la communication humaine. » (Goody, 
2006, p.20). Les trois aspects de cette notion nous intéressent pour asseoir notre méthodologie d'analyse de ce qui est dit et écrit à propos de l'événement sportif :

1. Lorsque le mot représente l'objet (le bassin de $50 \mathrm{~m}$ est un bassin qui existe concrètement et mesure précisément $50 \mathrm{~m}$ ),

2. Lorsque les mots parlent de l'action, ne représentant pas l'action elle-même mais sa réédition (gagner est la réédition de toucher le premier la plaque électronique à l'arrivée) (Goody, 2006, p.19),

3. Lorsque les mots s'éloignent des objets ou des actions pour aller vers les commentaires à propos de ce qui se passe, s'éloignant ainsi des principes de similitudes, de mimesis, d'imitation incluse dans la mimesis ("sortir l'affectif de tout cela» est un commentaire du DTN) (Goody, 2006, p.22).

Selon nous, les deux premiers aspects participent au réalisme de ce qui est dit ou écrit à propos de l'événement sportif alors que le troisième aspect s'éloigne du réalisme des faits. Les actions des sportifs qui vont se trouver en situation de compétition auront lieu au moment de l'effectuation de l'événement (Ouvrard-Servanton, 2010). Or, les événements sportifs vont se dérouler dans un espace-temps nommé Aiôn. À partir du lancement d'un événement, les expériences vont être en partie mémorisées à travers une mise en forme et en signes (Eco, 1992). Notre analyse se base sur les signes laissés dans les journaux c'est-àdire tout ce qui est produit en textes et images à propos de l'événement avant et après que les actions de l'événement s'effectuent. La question du signe est discutée en sémiotique. Umberto Eco distingue l'élément de la forme (type) et l'élément de la substance (token). Dans notre méthodologie, nous prenons le parti de considérer en premier lieu la forme comme élément apparent qui établit un rapport avec le contenu c'est-à-dire sa fonction sémiotique (Eco, 1992, p.12).

La deuxième considération découle de la première. L'événement sportif, artefact annoncé s'étalant dans l'Aion et se mémorisant à travers des signes, rassemble une série d'événements en ricochet: des petits et des grands événements. Ce phénomène de ricochet montre qu'il y a des temps notables et marqués. Par exemple, si deux nageurs appuient en même temps sur la touche électronique au centième de seconde près, comme aux CM de Shanghaï pour la course du 100 mètres dos, cet événement est remarquable parce que rare. Il y a aussi des temps en mouvement qui échappent à la mémorisation : les actions se font, les décisions se prennent, les échanges ont lieu sans que ces actions, ces décisions, ces échanges soient notées ou mémorisées ou tracées sous forme de signes. Henri Bergson l'aborde dans Matière et mémoire à propos de la durée (Bergson, 1965). L'événement sportif se développe dans la durée (celle de l'Aion) et les signes ne sont parfois que des images fixes ponctuant le déroulement de l'événement avec des coupures dans le temps qui échappent à la mémorisation traçable. Nous supposons alors que seuls les petits ou grands événements marquants sont les points singuliers et remarquables enregistrés dans des traces communicationnelles.

Les traces qui nous restent peuvent être classées et organisées et deviennent les points temporels que nous pouvons investiguer pour pouvoir retracer les événements. Ces traces sont des signes qui ont formes et contenus et dans lesquelles subsistent la mémoire de ce qui s'est passé et les prémisses de ce qui va advenir. Ceci reviendrait à dire que chaque événement sportif, artefactuel à l'origine, est une production à la fois passée et future. La communication autour des événements sportifs, l'information diffusée autour de ces événements et la production de représentations englobent un avant et un après. Il y a là production d'une histoire de l'événement qui a lieu dans le temps. Nous avons admis que 
la mise en scène (initiée par l'annonce d'un artefact: Championnats du Monde, CM, Championnats de France, CF) est ce qui déploie l'événement du passé vers le présent, en accumulant des séries d'événements, qui tire l'événement de communication remarquable du présent vers le passé. De même, nous avons admis que la mise en mémoire (à travers les textes et images laissés sur Internet et dans la presse papier) est ce qui s'étire après l'événement de communication et le tire du présent vers le futur. Dans la pratique et dans l'action, nous convenons que la mise en scène et la mise en mémoire sont enchevêtrées car il y a une utilisation de ce qui est mis en mémoire avant, pour la mise en scène, et une utilisation de la mise en scène pour effectuer la mise en mémoire.

Une partie de la communication est tracée, organisée et structurée dans un espace strié et une autre partie échappe à la structure, au prévu et au prévisible dans un espace lisse.

Nous avons choisi les huit numéros de L'Équipe suivants, pour les CM à Shanghai, en juillet 2011 : les 22, 23, 25, 27, 29, 30 et 31 juillet et les cinq numéros de L'Équipe suivants pour les CF à Dunkerque, en mars 2012 : les 19, 22, 23, 24, 25 mars. Pour la présente étude, nous avons sélectionné les articles lorsqu'ils mentionnaient dans leurs colonnes au moins un des éléments suivants: la FFN, la DTN, le nombre de nageurs sélectionnés, les temps de qualification pour les JO, les médailles.

\section{L'EVENEMENT SPORTIF : SIMULACRE OU REALITE?}

Les CM de Shanghai ou les CF de Dunkerque deviennent des événements publics dès que la décision et l'appellation, impliquant son organisation, sont annoncées. Nous considérons ce démarrage, non péjorativement, comme un simulacre qui existe dans la représentation avant même que la vérification de l'événement ait lieu dans et par l'action. Ce simulacre pourrait tomber dans la facétie et l'illusion (Jeanneret, 2000). Que diraient les médias si une de ces compétitions, pour une raison quelconque (une rupture politique ou environnementale ou économique, par exemple), n'avait pas lieu. Ceci pour souligner que tant que l'action n'a pas lieu et tant qu'elle n'est pas rendue visible publiquement, l'événement est un simulacre. On pourrait considérer que la seule preuve de la réalité d'un événement est sa réalisation. Or, nous ajoutons qu'en plus d'être «agi », l'événement «CF de Dunkerque »doit être rendu visible, donc public en étant notamment relayé par les médias. Dans la méthodologie, l'analyse sémiologique de l'organisation des signes est nécessaire mais non suffisante pour déterminer la réalité de l'événement sportif relayé, notamment par la presse écrite, et son rapport au cadre réglementaire. Il nous faudra mailler les signes avec d'autres composantes pour apporter les preuves de cette réalité.

Comme le souligne Yves Jeanneret (Jeanneret, 2000), un travail minutieux et laborieux est indispensable pour étudier un premier aspect : les conditions de publication médiatique, en tant que données sémiologiques. Puis, la question du statut est le second aspect à analyser car, de notre point de vue, c'est à ce stade qu'il peut y avoir abus d'influence d'un spécialiste ayant un point de vue personnel, sur la communication publique et sur le public. Afin que la valeur informative soit attribuée pour un fait réel, nous vérifions l'utilisation de références avérées et la traçabilité des enjambements de traduction dans la mise en discours des faits. Le troisième aspect est celui de la prise en compte du public lecteur : est-il un spécialiste? A-t-il suffisamment de culture en la matière pour pouvoir juger de la véracité du discours? Son rôle est-il de devenir l'arbitre d'un domaine qui l'intéresse sans pour autant avoir toutes les clés d'une décision arbitrale? Le quatrième aspect concerne l'analyse des écarts existants entre les discours tenus dans un cadre institutionnel et la vulgarisation de ces discours dans les médias. Il est donc nécessaire 
d'analyser la façon dont sont relayées les querelles entre les spécialistes sportifs, et pour finir, de prendre en compte des espaces sociaux hétérogènes, les sportifs, l'encadrement technique et institutionnel, les médias, les organisateurs, les sponsors, le public sur place et le public lecteur ou téléspectateur... Enfin, il faut également prendre en compte que les cultures sportives selon les pays, ont chacune leurs normes, qui sont parfois contradictoires. Le cinquième aspect, issu de l'anthropologie des techniques (Latour, 2001), nous incite à examiner l'enchevêtrement des acteurs humains et non humains participant à la fabrication des faits et à leur devenir événementiel. Le maillage de l'analyse de ces données laisse présumer d'une réalité des faits sportifs et permet de déterminer s'il y a glissement ou non dans les représentations au travers de la presse écrite diffusant l'information institutionnelle.

\section{L'INFLUENCE ET LA VISIBILITE DU CADRE INSTITUTIONNEL}

Nous avons croisé l'analyse du discours du DTN questionné, représentant le cadre réglementaire, et des articles de L'Équipe à partir des éléments présentés dans notre méthodologie avec des éléments quantitatifs (médailles gagnées et nombre de nageurs en lice). Cette analyse nous permet de vérifier l'intérêt des médias pour le caractère réaliste de l'événement (H2). Elle nous permet aussi d'examiner, à partir des éléments choisis, la façon dont la presse écrite contribue ou non à la visibilité de l'institution.

Dans une des réponses au questionnaire soumis en mai 2012, Claude Fauquet explique que l'instauration des critères de sélection ont changé en 1997 suite à l'échec des Jeux Olympiques d'Atlanta (1996). Précisément, deux points changent: les temps de sélection sont établis "en fonction de la réalité internationale» (et non plus fixés sur le niveau français) et ils doivent être réussis par les nageurs «à une date donnée, sans possibilité de rattrapage ». Les chiffres donnés par Claude Fauquet montrent l'ampleur de l'échec car sur les trente nageurs présents aux JO d'Atlanta, la France compte seulement quatre finalistes et sa meilleure place dans la compétition est quatrième. Or, après l'instauration des minima aux CM à Perth en 1998, seuls neuf nageurs sont qualifiés mais ils reviennent avec quatre médailles dont un titre mondial et trois de vices champions du monde. On peut noter que lors d'une compétition intermédiaire, les Championnats d'Europe de Séville en 1997, les changements dans les résultats sont notables : seize nageurs sélectionnés (le nombre de nageurs présents à la compétition baisse) et six médailles gagnées. Des résultats plus spectaculaires, dus à une proportion plus élevée entre le nombre de nageurs présents et les titres remportés, deviennent visibles aux JO d'Athènes car les vingt-quatre nageurs présents reviennent avec «six médailles dont un titre olympique ». Nous pouvons admettre que l'instauration des minima influence les nageurs dans leur entrainement, leurs objectifs et leurs résultats. En plus de peser sur les performances des nageurs, l'influence se situe à l'intérieur de la Fédération qui connait "beaucoup de perturbations ". Claude Fauquet a «pris la responsabilité de faire le tour de l'ensemble des régions françaises pour exposer et expliquer les raisons » de ces décisions. Il note que l'influence à l'extérieur de la Fédération a été "un intérêt médiatique, un regard nouveau de nos adversaires, un intérêt de nombreux DTN d'autres fédérations qui se sont inspirés de cette culture de l'exigence. ». Mais l'influence ne s'arrête pas là. Elle ne porte pas seulement sur les médias, sur la politique interne et sur le sport de haut niveau en France. L'influence a une incidence économique avec l'alliance avec «de nouveaux partenaires» et une incidence sur la visibilité nationale de la Fédération avec «une écoute plus importante de l'État». Elle aussi renforce les liens de la Fédération avec la base grâce à « une adhésion des 
Conseillers Techniques /CT» et «un changement de culture fédérale ». L'influence est aussi technique puisqu'un groupe d'entraineurs «a pris confiance et engagé de nombreuses avancées dans le domaine de l'entrainement». Elle devient internationale car la Fédération reçoit «beaucoup plus d'écoute et après une période d'observation de nombreux pays se sont inspirés de [ce] modèle. $»^{4}$.

La notion d'influence, comme décrite dans l'article d'Olivier Arifon (Arifon, 2008) dans son rapport à la communication dans un environnement international peut être transposée au contexte du rapport de la FFN et des médias. Les stratégies d'influence reposent sur un dialogue entre deux acteurs. Parfois il y a contournement d'un tiers acteur afin de pouvoir, le plus directement possible, sensibiliser et conquérir «l'opinion publique avec comme ressource, l'utilisation méthodique des médias» (Arifon, 2008). Le but est d'acquérir une crédibilité auprès du public. Il reste à vérifier en quoi le fait de rendre visible ou invisible aux yeux du public l'instauration de critères de sélection pour les compétitions internationales peut susciter un intérêt des lecteurs de la presse écrite et participer à la mise en ouvre de l'événement sportif et communicationnel. Cette interrogation porte sur plusieurs dimensions :

1. En partant de l'hypothèse émise par Françoise Papa que, désormais les Jeux Olympiques sont construits comme un événement sportif médiatisé (Papa, 1998, p.1) comment l'influence de l'information fournie par la FFN apparaît-elle dans la communication du média presse accessible au grand public?

2. Cette influence est-elle explicite et provoque-t-elle une déviation entre le cadre fixé, édité sous forme de document officiel, par la FFN et la façon dont la presse écrite utilise des formes communicationnelles pour relayer cette information ?

3. Quel intérêt porte la presse écrite à la création de l'événement sportif par la FFN grâce à son cadre officiel ? Ce cadre officiel est-il mis en valeur ou est-il un obstacle?

Wayne Brekhus se méfie des lieux d'enquête de nature publique qui « ont bien des chances de renvoyer des images de la vie sociale qui sont déjà les plus voyantes » (Brekhus, 2005, p.259). Il propose un renversement de marquage "comme à une stratégie explicite consistant à mettre en avant le non-marqué comme s'il était inhabituel et à ignorer le marqué comme s'il était ordinaire. » (Brekhus, 2005, p.260) car «comme tel, il constitue une manière efficace de problématiser certains éléments de notre monde qui vont de soi et de les rendre plus « visibles ».»(Brekhus, 2005, p.261).

Pour répondre aux interrogations sus-mentionnés et aux questions préalablement posées, nous avons repéré dans le corpus sélectionné, les éléments suivants afin de les marquer et de les rendre visibles :

a) Quand, comment et combien de fois sont cités la FFN, la DTN et les temps de qualification olympiques?

b) Le nombre de nageurs en lice et le nombre de médailles sont-ils mentionnés ?

${ }^{4}$ Questionnaire soumis par Marie Ouvrard-Servanton, par mail le 5 avril 2012 - Réponses reçues de Claude Fauquet, par mail, le 7 mai 2012. 
c) Quels sont les indicateurs sémiologiques, les écarts de référence et de statut, les mentions aux querelles et à l'hétérogénéité des cultures dans la communication médiatique ? Cette mise en scène représente-t-elle l'information institutionnelle?

\section{UNE ANALYSE ENTRE OMISSION ET POLEMIQUE}

\section{Les championnats du monde de Shanghai 2011}

Concernant les CM de Shanghai, parmi les quatre-vingt-treize articles qui ont été analysés, quatre datent du 22 juillet 2011, quatre du 23 juillet, huit du 24 juillet, dix-huit du 25 juillet, quinze du 27 juillet, quinze du 29 juillet, quatorze du 30 juillet, et quinze du 31 juillet 2011. Quatre-vingt-deux articles ont été laissés à l'écart pour la présente étude car ils ne mentionnaient pas les quatre éléments précités. Nous sommes bien conscients que ce que nous présentons ici sous forme de tableau est un résultat synthétisé que nous avons tenté de rendre le plus lisible possible malgré les limites de cette formalisation.

\begin{tabular}{|c|c|c|c|c|c|}
\hline $\begin{array}{l}\text { Date } \\
\text { L'Équipe }\end{array}$ & $\begin{array}{l}\text { FFN, DTN, } \\
\text { Médailles, } \\
\text { nombre } \\
\text { nageurs } \\
\text { sélectionnés }\end{array}$ & $\begin{array}{l}\text { Conditions de } \\
\text { publication }\end{array}$ & Statuts & $\begin{array}{l}\text { Querelles } \\
\text { Espaces culturels hétérogènes }\end{array}$ & $\begin{array}{l}\text { Enchevêtrement des } \\
\text { actants : acteurs } \\
\text { humains et non } \\
\text { humains }\end{array}$ \\
\hline \multirow[t]{2}{*}{$22 / 07 / 11$} & DTN \& $T^{2} S^{5}$ & $\begin{array}{l}\text { Rubrique : « Réactions } \\
\text { « au contrôle positif de } \\
\text { César Cielo, } 5 \text { lignes }\end{array}$ & $\begin{array}{l}\text { Technique \& } \\
\text { représentatif des } \\
\text { nageurs, } \\
\text { entraineurs, } \\
\text { Clubs ou pôles, } \\
\text { FFN. }\end{array}$ & $\begin{array}{l}\text { Querelle : } 4 \text { nageurs brésiliens } \\
\text { contrôlés positifs/la réglementation } \\
\text { FINA. } 4 \text { espaces culturelles : la FINA, } \\
\text { La Fédération brésilienne, I'avocat, les } \\
\text { nageurs, les médias, le public }\end{array}$ & $\begin{array}{l}\text { Au cœur de la } \\
\text { querelle: } \\
\text { Les nageurs (acteurs } \\
\text { humains) } \\
\text { La furosémide (acteur } \\
\text { non humain) }\end{array}$ \\
\hline & 21 nageurs & $\begin{array}{l}\text { Article } 1 / 8 \text { de page }+ \\
\text { photo } \\
\text { Noms des champions }\end{array}$ & $\begin{array}{l}\text { Nageurs } \\
\text { compétiteurs, } \\
\text { compétitions } \\
\text { internationales }\end{array}$ & $\begin{array}{l}\text { Querelle : entre différents types de } \\
\text { champions, ceux des grandes nations } \\
\text { et ceux des petites nations }\end{array}$ & $\begin{array}{l}\text { Les nageurs } \\
\text { compétiteurs (acteurs } \\
\text { humains) } \\
\text { Les médailles (acteurs } \\
\text { non humains) }\end{array}$ \\
\hline $23 / 07 / 11$ & $\begin{array}{l}\text { DTN, } \\
\text { Nombre } \\
\text { médailles }\end{array}$ & $\begin{array}{l}\text { Tableaux comparatifs } \\
\text { entre } 4 \text { nations: USA, } \\
\text { Australie, Chine, France } \\
\text { en médailles/nombre } \\
\text { de } \\
\text { licenciés/Bassins/Budge } \\
\text { ts }\end{array}$ & $\begin{array}{l}\text { Entraineurs } \\
\text { Fédérations } \\
\text { internationales, } \\
\text { Ministères des } \\
\text { sports }\end{array}$ & $\begin{array}{l}\text { Querelle : Force des résultats Faiblesse } \\
\text { des infrastructures }\end{array}$ & $\begin{array}{l}\text { Les entraineurs, } \\
\text { dirigeants, } \\
\text { représentants } \\
\text { nationaux (acteurs } \\
\text { humains) } \\
\text { Les bassins, l'argent } \\
\text { (acteurs non humains) }\end{array}$ \\
\hline $25 / 07 / 11$ & Médailles & $\begin{array}{l}\text { Obtention médailles de } \\
\text { bronze et d'argent } \\
\text { Photos, tableaux, } \\
\text { comparaisons avec } \\
\text { d'autres nageurs ou } \\
\text { nations. }\end{array}$ & $\begin{array}{l}\text { Nageurs, anciens } \\
\text { nageurs } \\
\text { Entraineurs } \\
\text { Commentateurs }\end{array}$ & $\begin{array}{l}\text { Querelle: Bronze obtenu/Or attendu } \\
\text { La culture sportive des différentes } \\
\text { nations }\end{array}$ & $\begin{array}{l}\text { Les nageurs } \\
\text { compétiteurs (acteurs } \\
\text { humains) } \\
\text { Les médailles (acteurs } \\
\text { non humains) }\end{array}$ \\
\hline $27 / 07 / 11$ & Médailles & 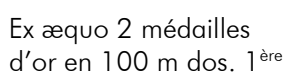 & $\begin{array}{l}\text { Nageurs, anciens } \\
\text { nageurs, }\end{array}$ & $\begin{array}{l}\text { Querelle : chronométrage natation aux } \\
\text { centièmes et pas aux millièmes }\end{array}$ & $\begin{array}{l}\text { Les nageurs } \\
\text { compétiteurs (acteurs }\end{array}$ \\
\hline
\end{tabular}

\footnotetext{
${ }^{5}$ TAS : Tribunal Arbitral du Sport

${ }^{6}$ FINA : Fédération Internationale de Natation
} 


\begin{tabular}{|c|c|c|c|c|c|}
\hline & & $\begin{array}{l}\text { fois } \mathrm{d}^{\prime} \text { or française } \\
\text { homme ef } 1 \text { ère fois } \\
\text { depuis création } \\
\text { mondiaux (en } 73 \text { ) : } 2 \\
\text { nageurs pour un titre }\end{array}$ & $\begin{array}{l}\text { Entraineurs, } \\
\text { Commentateurs }\end{array}$ & & $\begin{array}{l}\text { humains) } \\
\text { Les chronos (acteurs } \\
\text { non humains) }\end{array}$ \\
\hline 29/07/11 & Médailles & $\begin{array}{l}\text { Comparaison avec } \\
\text { I'Euro Budapest \& } \\
\text { autres mondiaux } \\
+ \text { comparaison } \\
\text { médailles } 100 \mathrm{~m} \text { avec } \\
\text { autres nations }\end{array}$ & Equipe de France & $\begin{array}{l}\text { Querelle : Entre nations } \\
\text { La culture sportive des différentes } \\
\text { nations }\end{array}$ & $\begin{array}{l}\text { Les nageurs } \\
\text { compétiteurs (acteurs } \\
\text { humains) } \\
\text { Les médailles (acteurs } \\
\text { non humains) }\end{array}$ \\
\hline $30 / 07 / 11$ & $\begin{array}{l}\text { FFN } \\
\text { DTN }\end{array}$ & $\begin{array}{l}\text { Retour de la nageuse } \\
\text { Laure Manaudou \& } \\
\text { Invitation }\end{array}$ & $\begin{array}{l}\text { Nageuse } \\
\text { individuelle, } \\
\text { championne de } \\
\text { natation la plus } \\
\text { titrée en France } \\
\end{array}$ & $\begin{array}{l}\text { Querelle : ancienne championne \& } \\
\text { probable championne }+ \\
\text { Française/entrainée aux USA }\end{array}$ & $\begin{array}{l}\text { Les nageurs } \\
\text { compétiteurs (acteurs } \\
\text { humains) }\end{array}$ \\
\hline 2 articles & $\begin{array}{l}\text { DTN } \\
\text { Médailles }\end{array}$ & $\begin{array}{l}\text { Choix des nageurs } \\
\text { relais } \\
\text { Bilan médailles de la } \\
\text { compétition }\end{array}$ & Nageurs, FFN & $\begin{array}{l}\text { Querelle : entre nageurs des différents } \\
\text { clubs } \\
\text { Les choix des différents clubs français }\end{array}$ & $\begin{array}{l}\text { Les nageurs } \\
\text { compétiteurs (acteurs } \\
\text { humains) } \\
\text { Les chronos, } \\
\text { médailles (acteurs non } \\
\text { humains) }\end{array}$ \\
\hline \multirow[t]{2}{*}{$31 / 07 / 11$} & Ex DTN & $\begin{array}{l}\text { Entretien avec un } \\
\text { entraineur national }\end{array}$ & $\begin{array}{l}\text { Relation } \\
\text { Entraineurs/nage } \\
\text { urs \& Esprit } \\
\text { fédération }\end{array}$ & $\begin{array}{l}\text { Querelle : Les clubs régionaux dans la } \\
\text { vague ou pas du national } \\
\text { La culture des différents clubs français }\end{array}$ & $\begin{array}{l}\text { Les nageurs } \\
\text { compétiteurs (acteurs } \\
\text { humains) }\end{array}$ \\
\hline & DTN & $\begin{array}{l}\text { Relations internes } \\
\text { équipe de France }\end{array}$ & $\begin{array}{l}\text { Entre les nageurs } \\
\text { ou groupes de } \\
\text { nageurs ou } \\
\text { entraineurs }\end{array}$ & $\begin{array}{l}\text { Querelle : Entre clubs régionaux } \\
\text { français et nageurs }\end{array}$ & $\begin{array}{l}\text { Les nageurs et } \\
\text { entraineurs (acteurs } \\
\text { humains) }\end{array}$ \\
\hline
\end{tabular}

Tableau 1 : Analyse des articles des CM de Shanghai, juillet 2011

$\mathrm{Au}$ travers des onze articles retenus, au regard des éléments répertoriés dans la première colonne du tableau, le DTN est mentionné six fois en début et en fin de compétition et la FFN une seule fois (a). Cette recherche du nombre de fois où la parole est donnée à une institution ou une personne, citées dans les médias, est ce que nous nommons allitérations médiatiques, un type (et non un token), indicateur de la place de cette institution ou de ce représentant institutionnel dans les médias. Le nombre de nageurs en lice n'est mentionné qu'une fois (b) alors que la référence au nombre de médailles est faite cinq fois dont deux fois par comparaison et une fois pour le bilan final de la compétition. Le rappel des temps de qualification nécessaires pour cette compétition et le lien avec la FFN n'est mentionné à aucun moment dans les articles. Ces éléments quantitatifs montrent que l'institution est représentée mais que le cadre réglementaire institutionnel est omis et confirme en partie notre troisième hypothèse (H 3).

Seuls trois articles (un du 30/07/11, deux du 31/07/11) sont centrés uniquement sur les acteurs humains et n'enchevêtrent pas les acteurs humains et non humains. A partir des autres indicateurs (c), nous pouvons montrer que ces articles sont construits autour de la querelle soit entre clubs régionaux français et le plan national soit autour des nageurs dans leur rapport à l'équipe de France. Dans les articles du 30 juillet 2011, le DTN exerce son rôle médiateur en relation avec les médias comme défini par son projet fédéral « externe ». Il invite une championne olympique française qui s'était éloignée de la compétition à rejoindre l'équipe de France pour son prochain stage. En tant que représentant de l'institution, il donne un poids réaliste à cette information, confirmant à la fois la première 
hypothèse (H1) qui mentionne que le cadre réglementaire érige de fait la compétition sportive qui sans lui n'aurait pas lieu d'être et la deuxième hypothèse (H2) mentionnant la nécessité du cadre réglementaire pour la presse écrite afin de rendre réaliste l'événement sportif. Puis, il est celui qui, après concertation avec les autres responsables de l'équipe de France, transmet l'information sur les nageurs présents aux relais. Sur les choix non prononcés par le DNT sur la composition d'un relais, l'envoyé spécial donne ses hypothèses et avis alimentant une forme polémique : «On a vu que la polémique dans toutes ses variétés discursives constitue une forme de communication «spectaculaire ». Dès lors qu'elle est médiatisée, il semble que la polémique révèle bien la tension paradoxale qui nourrit les cultures médiatiques contemporaines depuis des décennies. » (Amossy \& Burger, 2011, p. 9). Là, l'information institutionnelle est tirée vers le spectaculaire par l'omission d'éléments informatifs réalistes, infirmant notre deuxième hypothèse (H2).

Dans un des deux articles du 31 juillet, correspondant au dernier jour de la compétition, Claude Fauquet est cité, en tant qu'ami, ancien DTN et Directeur adjoint de l'INSEP, pour mentionner son opinion sur un entraineur d'un des Pôles France Jeunes, qu'il qualifie de discret, bien que deux de ses nageurs aient été médaillés. Cet événement médiatique créé sur l'événement sportif (événements en ricochet) montre l'ancien DTN qui admet être médiatique, mettant en lumière le travail d'un entraineur dont les nageurs viennent d'être médaillés (composante réaliste). Ici l'information institutionnelle contribue à l'aspect remarquable de l'événement sportif mis en exergue par le média. Ceci infirme la troisième hypothèse (H3) excipant que le média presse s'empare de ce qui se passe « hors cadre » en omettant la source de l'information institutionnelle pour promouvoir une communication événementielle.

Dans le deuxième article à cette date, l'hypothèse d'une mésentente entre les membres de l'équipe de France est soulevée mais non confirmée. "On guettait avec le DTN le moment où ça pourrait ne pas aller, mais ça ne s'est pas produit», témoigne le directeur des équipes. C'est un discours de l'ordre du commentaire où le lecteur n'est pas spécialiste. Ce discours ne fait référence ni à des éléments réalistes, comme des références avérées, ni à des éléments traçables dans la traduction à partir de la source de la querelle. Le média prend soin de citer sa parole pour rendre explicite que la polémique n'a pas eu lieu mais aurait pu avoir lieu. La communication médiatique aimerait aussi créer de l'événement en "remarquant»ce qui ne se réalise pas. Ce non réalisme de l'événement médiatique infirme à nouveau la deuxième hypothèse (H2) qui pose comme un a priori que la presse écrite a besoin du cadre institutionnel pour rendre réaliste l'événement sportif. Que la querelle soit polémique ou controverse, ces trois articles n'ont pas de racines dans l'action réelle. Ils relèvent plus du spectaculaire sachant que «le caractère spectaculaire des discours polémiques permet aussi d'embrayer de manière optimale la logique commerciale à l'œuvre dans les médias. ». (Amossy \& Burger, 2011, p. 10). Pour ce deuxième article, ces caractéristiques infirment à nouveau la deuxième hypothèse (H2) et confirment que le média presse s'empare de ce qui se passe « hors cadre » en rendant invisible la source de l'information institutionnelle pour favoriser une communication événementielle (H3).

Dans l'article du 23 juillet 2011, la DTN est citée en référence au changement que Claude Fauquet a fait et rendu public en 1997. Ce changement est médiatisé ainsi : "Ce qui a permis à la France de grandir, ce sont les critères de sélection sévères instaurés par Claude Fauquet (Directeur des équipes de France de 1995 à 2000 puis DTN jusqu'en 2008). Ça a été une révolution ». Bien que la date du changement soit omise, la référence explicite au cadre et à l'information institutionnelle infirme la troisième hypothèse (H3). Le journal se 
sert spécifiquement de l'information institutionnelle pour montrer le bien-fondé de l'événement sportif.

Pour les autres articles, en associant les éléments relevés dans les conditions de publication (c) et la façon dont les actants sont enchevêtrés, autant pour la question du dopage que pour les comparaisons de performance entre nations ou entre nageurs ou que pour l'obtention de médailles, les références mentionnées par les articles sont avérées, c'est-àdire reconnues par tous (spécialistes et non spécialistes) ce qui confère un caractère réaliste à l'événement (H2).

Concernant le dopage et les articles qui le mentionnent, le public lecteur ne peut pas jouer le rôle d'arbitre. Il n'a pas de statut de spécialiste. Il n'est ni expert en biochimie, pour la composition des substances dopantes, ni expert en droit, pour juger d'une peine. Il peut être sensible au rapport compétition et «tricherie » car il peut avoir besoin d'admirer des vainqueurs qui aient de la valeur et avoir une opinion à ce sujet, mais est pris en otage dans cet aspect événementiel.

Nous avons tenu compte des médailles car dans le document réglementaire de la FFN pour les qualifications, les médaillés des CM de Shanghaï peuvent être sélectionnés en priorité pour les Championnats d'Europe en petit bassin de décembre 2011. Néanmoins, cette partie de l'information n'est pas relayée par la presse lors des CM de Shanghaï. Dans ce cas, la communication médiatique sur les médailles gagnées omet l'information institutionnelle, confirmant la troisième hypothèse (H3).

\section{Les championnats de France de Dunkerque 2012}

Concernant les soixante-treize articles analysés pour les CF de Dunkerque, dix-sept articles datent du 19 mars 2012, seize du 22 mars, dix-sept du 23 mars, quatorze du 24 mars et neuf du 25 mars. Neuf d'entre eux ont été retenus puisqu'ils mentionnaient les quatre éléments sélectionnés comme éléments de forme dans les signes médiatiques.

\begin{tabular}{|l|l|l|l|l|l|}
\hline $\begin{array}{l}\text { Date } \\
\text { L'Équipe }\end{array}$ & $\begin{array}{l}\text { FFN, DTN, } \\
\text { Médailles, } \\
\text { nombre } \\
\text { nageurs } \\
\text { sélectionnés } \\
\text { Temps de } \\
\text { qualification }\end{array}$ & Conditions de publication & Statuts & $\begin{array}{l}\text { Querelles } \\
\text { Espaces culturels } \\
\text { hétérogènes }\end{array}$ & $\begin{array}{l}\text { Enchevêtrement des } \\
\text { actants : acteurs } \\
\text { humains et non } \\
\text { humains }\end{array}$ \\
\hline 19/03/12 & $\begin{array}{l}\text { Temps de } \\
\text { qualification }\end{array}$ & $\begin{array}{l}\text { Rappel sous forme de listes } \\
\text { regroupées : en individuel, en } \\
\text { relais, Hommes, Femmes }\end{array}$ & $\begin{array}{l}\text { Tous les nageurs } \\
\text { \& entraineurs } \\
\text { Equipe de France }\end{array}$ & $\begin{array}{l}\text { Querelles : entre nombre } \\
\text { de nageurs et temps de } \\
\text { qualification }\end{array}$ & $\begin{array}{l}\text { Nageurs compétiteurs } \\
\text { et entraineurs (acteurs } \\
\text { humains) } \\
\text { Distance (acteur non } \\
\text { humain) }\end{array}$ \\
\hline qualification & $\begin{array}{l}\text { Nageuse citée 2 fois en début et } \\
\text { fin de colonne + citation } \\
\text { personnelle }\end{array}$ & $\begin{array}{l}\text { La première } \\
\text { nageuse } \\
\text { sélectionnée pour } \\
\text { les JO dans cette } \\
\text { compétition }\end{array}$ & $\begin{array}{l}\text { Querelle : entre les } \\
\text { espérés qui ratent et ceux } \\
\text { qui réussissent }\end{array}$ & $\begin{array}{l}\text { Nageuse (acteur } \\
\text { humain) et affiche, } \\
\text { chambre (acteurs non } \\
\text { humains) }\end{array}$ \\
\hline 22/03/12 & $\begin{array}{l}\text { Temps de } \\
\text { qualification }\end{array}$ & $\begin{array}{l}\text { 2 rappels : un des temps exigés et } \\
\text { un des qualifiés. 2 mini encarts } \\
\text { pour 2 nageurs Champions } \\
\text { Olympiques et 4 citations de } \\
\text { nageurs (photographiés), une } \\
\text { mention de rappel dans un long } \\
\text { article, un rappel de la règle de } \\
\text { qualification pour le 100 m }\end{array}$ & $\begin{array}{l}\text { Nageurs } \\
\text { compétiteurs ou } \\
\text { choix éditorial du } \\
\text { journal }\end{array}$ & $\begin{array}{l}\text { Querelles : Compétition } \\
\text { interne à l'Équipe de } \\
\text { France et entre les Clubs } \\
\text { ou Pôles France }\end{array}$ & $\begin{array}{l}\text { Nageurs compétiteurs } \\
\text { et entraineurs (acteurs } \\
\text { humains) } \\
\text { Lignes d'eau (acteur } \\
\text { non humain) }\end{array}$ \\
\hline
\end{tabular}




\begin{tabular}{|c|c|c|c|c|c|}
\hline $23 / 03 / 12$ & $\begin{array}{l}\text { Temps de } \\
\text { qualification }\end{array}$ & $\begin{array}{l}\text { Rappel de la réglementation pour } \\
\text { le relai par l'entraineur du } \\
\text { Champion Olympique } \\
2 \text { rappels: un des temps exigés et } \\
\text { un des qualifiés }\end{array}$ & Entraineur & $\begin{array}{l}\text { Querelle : entre ceux qui } \\
\text { veulent respecter la règle } \\
\text { instaurée par la FFN } \\
\text { depuis } 1997 \text { et les autres }\end{array}$ & $\begin{array}{l}\text { Nageurs compétiteurs } \\
\text { et entraineurs (acteurs } \\
\text { humains) } \\
\text { Table de massage } \\
\text { (acteur non humain) }\end{array}$ \\
\hline & $\begin{array}{l}\text { Temps de } \\
\text { qualification } \\
\text { Médaille }\end{array}$ & $\begin{array}{l}\text { Citations ou commentaires de } 3 \\
\text { nageurs qualifiés et } d^{\prime} \text { un ancien } \\
\text { médaillé }\end{array}$ & $\begin{array}{l}\text { Nageurs } \\
\text { compétiteurs }\end{array}$ & $\begin{array}{l}\text { Querelle : entre ceux qui } \\
\text { veulent respecter la règle } \\
\text { instaurée par la FFN } \\
\text { depuis } 1997 \text { et les autres }\end{array}$ & $\begin{array}{l}\text { Nageurs compétiteurs } \\
\text { (acteurs humains) } \\
\text { Grand bassin, } \\
\text { distance, place } \\
\text { (acteurs non humains) }\end{array}$ \\
\hline \multirow[t]{2}{*}{$24 / 03 / 12$} & $\begin{array}{l}\text { Temps de } \\
\text { qualification }\end{array}$ & $\begin{array}{l}\text { Référence aux qualifications } \\
\text { précédentes aux JO et } \mathrm{CM} \\
2 \text { rappels : un des temps exigés et } \\
\text { un des qualifiés }\end{array}$ & $\begin{array}{l}\text { Nageurs } \\
\text { compétiteurs }\end{array}$ & $\begin{array}{l}\text { Querelle : entre les déjà } \\
\text { qualifiés toujours en } \\
\text { compétition, les qualifiés } \\
\text { potentiels et les non } \\
\text { qualifiés }\end{array}$ & $\begin{array}{l}\text { Les nageurs } \\
\text { compétiteurs (acteurs } \\
\text { humains) } \\
\text { Chronos (acteurs non } \\
\text { humains) }\end{array}$ \\
\hline & DTN & $\begin{array}{l}\text { Organisation d'une cérémonie } \\
\text { avec sélectionnés olympiques mais } \\
2 \text { absents autorisés par leur } \\
\text { entraineur }\end{array}$ & $\begin{array}{l}\text { FFN, Équipe de } \\
\text { France }\end{array}$ & $\begin{array}{l}\text { Querelle : ceux qui } \\
\text { donnent de l'importance } \\
\text { aux cérémonies publiques } \\
\text { et ceux qui n'en donnent } \\
\text { pas }\end{array}$ & $\begin{array}{l}\text { Nageurs } \\
\text { compétiteurs, } \\
\text { entraineurs, DTN, } \\
\text { couturières (acteurs } \\
\text { humains) } \\
\text { Photo (acteur non } \\
\text { humain) }\end{array}$ \\
\hline \multirow[t]{2}{*}{$25 / 03 / 12$} & Médailles & $\begin{array}{l}2 \text { rappels: un des temps exigés et } \\
\text { un des qualifiés } \\
\text { Rappel des médailles du } \\
\text { champion olympique du } 100 \mathrm{~m}\end{array}$ & $\begin{array}{l}\text { Champion } \\
\text { olympique }\end{array}$ & $\begin{array}{l}\text { Absence de querelle : } \\
\text { Unanimité autour de la } \\
\text { reconnaissance d'un } \\
\text { grand champion }\end{array}$ & $\begin{array}{l}\text { Champion olympique } \\
\text { (acteur humain) } \\
\text { Médailles (acteurs } \\
\text { non humains) }\end{array}$ \\
\hline & DTN & $\begin{array}{l}\text { Laisser sa place pour la } \\
\text { qualification aux JO }\end{array}$ & $\begin{array}{l}\text { Nageuses } \\
\text { compétitrices, } \\
\text { FFN }\end{array}$ & $\begin{array}{l}\text { Querelle : Entre ceux qui } \\
\text { veulent respecter la règle } \\
\text { instaurée par la FFN } \\
\text { depuis } 1997 \text { et les autres }\end{array}$ & $\begin{array}{l}\text { Champion olympique } \\
\text { (acteur humain) } \\
\text { Places (acteurs non } \\
\text { humains) }\end{array}$ \\
\hline
\end{tabular}

Tableau 2 : Analyse des articles des CF de Dunkerque, mars 2012

La DTN n'est citée que deux fois (a) pour deux événements de communication qui échappent aux cadres instaurés (ceux de la FFN, notamment), au prévu, au prévisible. Le premier événement est lorsque l'athlète, championne olympique, propose de laisser sa place de $1^{\text {ère }}$ à la $3^{\text {ème }}$ parce que "c'est l'esprit d'équipe », "si elle n'avait pas été en finale, je n'aurais sans doute pas nagé aussi vite ». Effectivement, la troisième a réussi le temps de qualification mais la deuxième condition est d'être dans les deux premières pour être qualifiée. Le DTN actuel, Christian Donzé, en profite pour rappeler le règlement et invite à sortir «de l'affectif» et du «buzz», en privilégiant «la réalité du haut niveau». Le surgissement de l'opinion d'une nageuse, opinion annoncée par elle, par le biais d'un média audiovisuel, puis reprise par le journal L'Équipe, bouscule l'information institutionnelle (le cadre réglementaire de la FFN) et donne l'occasion de réitérer la réglementation fédérale. Dans un premier temps, cet événement confirme notre troisième hypothèse (H3) puis l'infirme grâce au rôle pris par le DTN (rôle fédéral externe). Ceci revient à dire que simultanément le média presse écrite se saisit de ce qui se passe « hors cadre » en omettant la référence à l'institution pour favoriser la communication événementielle et donne une place à l'information institutionnelle en tant que telle en donnant la parole au DTN.

À nouveau, le deuxième événement confirme la troisième hypothèse (H3) et l'infirme par l'intervention du DTN, en bousculant le cadre de la FFN car deux athlètes qualifiés sur la 
plus haute marche de la compétition, pour les JO de Londres, ne seront pas présents lors de la cérémonie présentant officiellement les sélectionnés et manqueront pour la photo officielle. Leur entraineur les a autorisés à partir. Le journaliste signale que «le DTN est gêné mais ne veut pas polémiquer ». L'article est court et en bas de page.

Les autres articles (sept articles sur les neuf sélectionnés) mettent l'accent sur les qualifications et les médailles (b), à l'intérieur desquels les surprises et les déceptions des nageurs qualifiés ou non qualifiés sont largement exprimées. La référence au DTN et à la FFN et le nombre de nageurs en lice au niveau national sont omis bien que les temps de qualification soient directement issus du cadre de la FFN. Nous relevons ces éléments qui, ici, invalident la deuxième hypothèse (H2) et montrent que la presse écrite n'a pas besoin du cadre institutionnel pour créer le réalisme de l'événement sportif.

Dans les articles des CF de Dunkerque, il y a toujours des acteurs humains enchevêtrés à des acteurs non humains dans la fabrication de l'événement par la presse écrite prédisposant au réalisme des faits dans la communication médiatique (H2). Concernant les conditions de publication (c) lors des CF de Dunkerque, le journal réserve deux encarts, l'un nommé : «Qualification olympique, mode d'emploi » et l'autre «En route pour Londres, Qualifiés pour les Jeux ». Dans ces deux encarts, bien qu'aucune référence ne soit faite à la FFN ou à son document et que les informations soient synthétisées, nous pouvons vérifier que celles-ci sont justes. Malgré cela, le lecteur non spécialiste peut suivre et comprendre les conditions requises pour la sélection des nageurs aux JO de Londres. Cependant, par la carence de la source de l'information et le manque de références avérées, le lecteur est implicitement enclin à croire le journal puisque l'information institutionnelle disparait. Ce manque de représentation ou une sous-représentation du cadre fédératif en tant qu'institution entérine que le média presse écrite n'aurait pas besoin de l'information institutionnelle pour donner un caractère réaliste à l'événement (H2) et que ces omissions rendent invisible l'information institutionnelle au profit de la communication événementielle (H 3).

Bien qu'un rappel allitératif soit fait concernant les règles de qualification pour les JO, les querelles relayées par le journal L'Équipe sont majoritairement centrées sur ceux qui veulent respecter les critères établis depuis 1997 et ceux qui aimeraient ne pas appliquer la règle (trois articles). Néanmoins, l'échantillon des querelles est varié. On retrouve la polémique entre les différents clubs régionaux ou Pôles France et entre les nageurs y appartenant. En revanche, une nouvelle polémique apparaît concernant la présence des athlètes aux cérémonies officielles. En l'occurrence, la cérémonie mentionnée n'est pas anodine puisqu'elle permet de rassembler dans un même espace, dans un même moment puis sur une même photo, tous les nageurs français qui seront présents aux JO de Londres. Or, deux nageurs manquent à l'appel de cet événement. Un événement se greffe sur l'événement (l'événement s'effectue par ricochet dans l'Aiôn). Avant que la polémique ne gonfle, le DTN intervient pour signifier que ces athlètes étaient autorisés à s'absenter comme nous l'avons déjà signalé sur les critères d'analyse (a). Si le DTN mentionne que les nageurs sont soumis à une autorisation pour s'absenter, il sous- entend simultanément qu'il y a obligation d'être présent. Néanmoins, le journal saisit la balle au bond pour mettre en avant ce qui échappe à la règle. Ces éléments corroborent ceux analysés précédemment (a) confirmant H3 et l'infirmant par l'intervention du DTN.

Seul un article affiche l'unanimité pour saluer les performances d'un grand champion olympique qui n'a pas réussi sa sélection pour les Olympiades de Londres (sauf au relais). Une page entière lui est consacrée avec de nombreux témoignages et commentaires (c). Ce 
sont ses médailles et ses performances de sportif (a) qui créent l'événement et qui lui confèrent un caractère réaliste. Pour ce sportif, les petits et grands événements précédents (entrainements, chronos, courses gagnées et perdues, titres, etc.) qui ont été réalisés grâce à l'instauration d'un cadre institutionnel réglementaire et qui, par ricochet, créent cet événement ont une base réaliste (H2) puisque les courses ont été nagées, gagnées, perdues et médaillées dans le respect du cadre institutionnel (qui est omis et donc seulement implicite confirmant ainsi H3). Ils ont été préparés et mis en mémoire.

\section{CONCLUSION}

Nous pouvons conclure que notre première hypothèse $(\mathrm{H} 1)$ affirmant que le cadre réglementaire érige de fait la compétition sportive comme un artefact qui sans lui n'aurait pas lieu d'être, dessine une relation de condition entre les spécialistes d'un sport, ici les entraineurs de natation, les nageurs, la DTN, et le monde médiatique. En ce sens cette relation de condition crée un effet attendu qui est la compétition dont la publication du cadre réglementaire est irrévocablement nécessaire.

La deuxième hypothèse (H2) stipulant que la presse écrite a besoin du cadre réglementaire pour rendre réaliste l'événement sportif, est infirmée pour deux raisons essentiellement. La première est que la FFN n'est que très peu mentionnée comme étant la source de l'information institutionnelle alors que cette dernière garantit, en partie, le réalisme de l'événement et est un garde-fou pour ne pas aboutir à un simulacre, voire à une illusion des compétitions et des résultats. La deuxième raison est que les querelles sont omniprésentes bien que nous ayons a priori sélectionné des articles dans L'Équipe à partir d'éléments quantifiables et réalistes (mention de la FFN ou du DTN, du nombre de nageurs en lice, des médailles et des temps de qualification) pour écarter les autres articles concernant les mêmes compétitions.

En revanche, la deuxième hypothèse $(\mathrm{H} 2)$ est confirmée à deux reprises :

1. Quand le DTN représentant de la FFN rappelle que les règles de qualification sont devenues strictes et calées sur les niveaux internationaux. Alors, on voit que, pour un sport comme la natation, le nombre d'athlètes qualifiés et médaillés a augmenté, engendrant des événements en chaine. Le média presse relaie de façon notable les événements concernant les médailles gagnées lors des compétitions internationales et concernant les qualifications pour les JO lors de compétitions nationales.

2. Quand l'unanimité s'exprime autour d'un sujet: la reconnaissance d'un grand champion. Pour notre étude, ce contre-exemple de l'avènement d'un événement sans polémique nous amène à évoquer que la proportion des polémiques médiatiques satisfaisant des intentions commerciales est élevée. La presse est orientée vers l'événement mais parfois elle se base sur un événement sportif réaliste et avéré alors qu'à l'extrême opposé (dans l'article du 31 juillet de Shanghai) elle se base sur une inexistence de faits.

Quant à la troisième hypothèse (H3) alléguant que le média presse écrite s'empare de ce qui se passe «hors cadre » en rendant invisible la source de l'information institutionnelle pour favoriser une communication événementielle, substantiellement, les nombreuses omissions relevées concernant l'information institutionnelle dénotent que celle-ci est peu relayée par les médias (H3). Le DTN de la FFN est moins cité dans la compétition nationale choisie que dans la compétition internationale. Ce cadre est-il minimisé car considéré seulement comme une condition préalable qui va de soi ? Nous pouvons noter 
que les deux fois où la presse écrite s'empare de la polémique, la sollicitation arrive d'une nageuse ou de la DTN. Concernant la proposition d'une nageuse olympique de remettre éventuellement en cause le cadre instauré par la FFN, cet événement imprévu n'est pas anodin puisque c'est la nageuse la plus titrée de tous les temps en natation française. L'abus de privilèges que nous avons mentionné en amont est tentant: bousculer le cadre officiel en passant par la communication médiatique.

L'écart entre le document mis en ligne par la FFN, information institutionnelle, et l'information retranscrite par le journal est moindre. En revanche, l'institution n'est que peu présentée et peu représentée (confirmation de H3). Sa représentation existe par les mots du DTN quand il prend le temps d'expliquer le cadre de la FFN, notamment lorsque le cadre est bousculé par certains sportifs de haut niveau qui aimeraient réinventer les règles. Le fait que l'information institutionnelle, qui cadre la compétition, soit rendue visible pourrait-il permettre aux journalistes de faire l'éloge de ce cadre qui, sans lui, ne permettrait pas à l'événement de se réaliser, tout en laissant un espace d'improvisation?

En résumé, pour répondre à notre question principale, deux points retiennent notre attention :

1. D'une part, lorsque le média presse écrite retransmet l'information officielle de la Fédération de natation telle que la réglementation des compétitions, il ne mentionne pas la Fédération comme source alors que l'événement sportif qui devient communicationnel et médiatique ne peut exister sans la réglementation issue des fédérations. L'omission peut ressembler à un «allant de soi ». L'information institutionnelle sur la réglementation est donnée. C'est un «donné ». Nous n'avons pas exploré les hypothèses interprétatives concernant les intentions du journal d'omettre la source. Présuppose-t-il que la référence aux sources est négligeable ? Ou présuppose-t-il que la source ne peut intéresser le lecteur? Le média veut-il se substituer à l'institution publique dans la communication publique?

2. D'autre part, l'analyse confirme que l'événement médiatique a souvent lieu lorsque les sportifs ou un des acteurs spécialistes sortent du cadre donné et créent l'inattendu. Le journaliste cherche ce qui sort de l'ordinaire et va vers ce qui troue la logique de l'évidence. De notre point de vue, le journaliste met en avant ce qui se passe à travers le maillage de l'espace strié pour aller vers l'espace lisse. Alors, ce qui marque, est le spectaculaire qui devient visible au public.

L'information institutionnelle de la FFN s'adresse prioritairement aux spécialistes et la communication médiatique est plutôt destinée au grand public. Dans la production de l'événement, la fédération sportive a besoin du cadre réglementaire alors que le média presse écrite a besoin d'attirer l'attention d'un public le plus vaste possible. L'information institutionnelle nourrit la communication médiatique et les médias s'en servent pour mettre en exergue ce qui échappe aux cadres instaurés. L'information institutionnelle confère un réalisme à l'événement sportif communiqué par les médias qui rendent publique l'information institutionnelle tout en omettant très souvent de nommer l'institution. L'information institutionnelle ne semble pas être intéressante commercialement. Cependant, dans le cas de la Fédération Française de Natation, l'institution par le biais de ses représentants se réserve la possibilité d'intervenir dans la communication médiatique particulièrement quand les faits débordent et peuvent entraver le bon déroulement des compétitions. Dans ces moments-là, la FFN est mise en lumière pour rappeler les règles à suivre. 


\section{REFERENCES BIBLIOGRAPHIQUES}

Agostinelli, Serge. (4/2009). Comment penser la médiation inscrite dans les outils et leurs dispositifs: une approche par le système artefactuel. Distances $\mathcal{E}$ Savoirs, 7, p.355-376.

Agostinelli, S. (2003). Les nouveaux outils de communication des savoirs. Paris : L'Harmattan, Éd.

Amossy Ruth \& Burger, Marcel, «Introduction : la polémique médiatisée », Semen [En ligne], 31 |2011, mis en ligne le 01 avril 2011, consulté le 01 septembre 2012. URL: http://semen.revues.org/9072

Arifon, Olivier. (2008). Influence et communication dans l'environnement international: le cas de la diplomatie non gouvernementale. Récupéré sur http://halshs.archives-ouvertes.fr: http://archivesic.ccsd.cnrs.fr/sic 00466595

Arquembourg, J. (2010) "Des images en action », Réseaux 5/2010 (n 163), p. 163-187. URL : www.cairn.info/revue-reseaux-2010-5-page-163.htm. DOI : $\underline{10.3917 / \text { res.163.0163. }}$.

Bergson, Henri. (1939/1965). Matière et mémoire. Paris: P.U.F.

Brekhus Wayne, «Une sociologie de l'« invisibilité » : réorienter notre regard », Réseaux, 2005/1 n 129-130, p. 243-272. DOI : 10.3917/res.129.0243

Deleuze, G. (1969). La logique du sens. Paris: Les Editions de Minuit.

Deleuze, G., \& Guattari, F. (1980). Mille plateaux. Paris: Les Éditions de Minuit.

Eco, Umberto (1992). La production de signes. Paris: Librairie Générale Française.

Goody, Jack. (2006). La peur des représentations. Paris: Editions La Découverte.

Jeanneret, Yves. (2000). La trivialité comme évidence et comme problème: à propose de la querelle des impostures. Enjeux de l'Information et de la Communication. [en ligne] http://w3.u-grenoble3.fr/les enjeux/2000/Jeanneret/index.php

Latour, B. (2001). L'espoir de Pandore. Paris: La Découverte.

Lavoinne, Yves. (1997). Le langage des médias. Grenoble: Presses Universitaires de Grenoble.

Ouvrard-Servanton, Marie. (2010). Regard SIC sur le rôle des actants - Anthropologie d'un événement de communication dans une organisation à visée internationale. Thèse de doctorat, Aix-Marseille Université, 311 p.

Papa, Françoise. (1998, Octobre). Jeux Olympiques: du signal universel à la pluralité des images. Communication $n^{\circ} 67$. 